\title{
A novel similarity score based on gene ranks to reveal genetic relationships among diseases
}

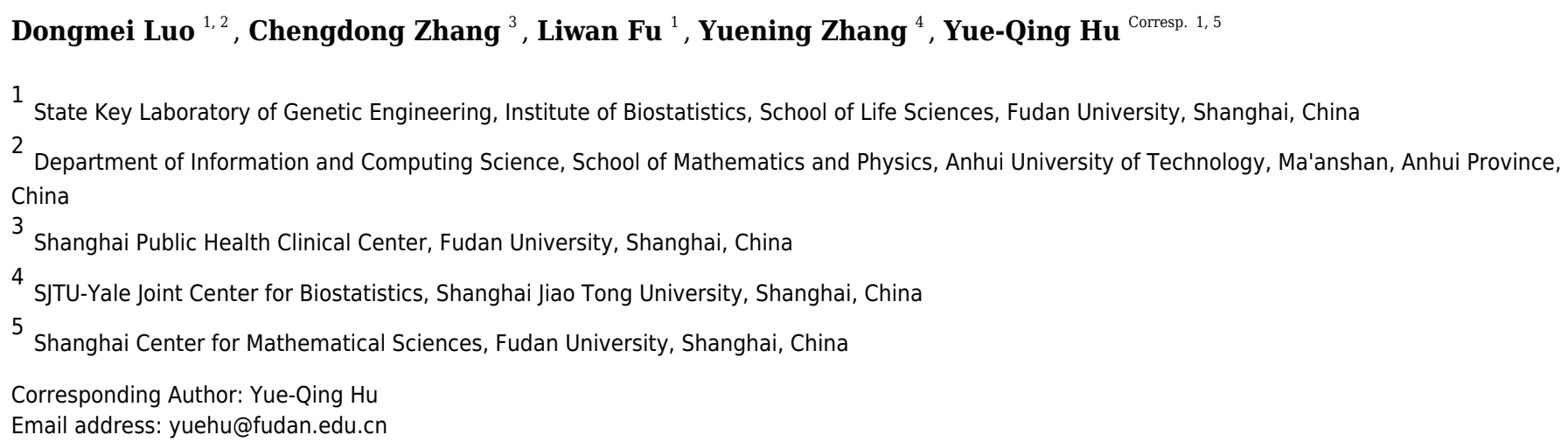

Knowledge of similarities among diseases can contribute to uncovering common genetic mechanisms. Based on ranked gene lists, a couple of similarity measures were proposed in the literature. Notice that they may suffer from the determination of cutoff or heavy computational load, we propose a novel similarity score SimSIP among diseases based on gene ranks. Simulation studies under various scenarios demonstrate that SimSIP has better performance than existing rank-based similarity measures. Application of SimSIP in gene expression data of 18 cancer types from The Cancer Genome Atlas shows that SimSIP is superior in clarifying the genetic relationships among diseases and demonstrates the tendency to cluster the histologically or anatomically related cancers together, which is analogous to the pan-cancer studies. Moreover, SimSIP with simpler form and faster computation is more robust for higher levels of noise than existing methods and provides a basis for future studies on genetic relationships among diseases. In addition, a measure MAG is developed to gauge the magnitude of association of anindividual gene with diseases. By using MAG the genes and biological processes significantly associated with colorectal cancer are detected. 


\title{
A novel similarity score based on gene ranks to reveal genetic relationships among diseases
}

\author{
Dongmei Luo ${ }^{1,2}$, Chengdong Zhang ${ }^{4}$, Liwan Fu ${ }^{1}$, Yuening Zhang ${ }^{5}$, and \\ Yue-Qing $\mathrm{Hu}^{1,3}$
}
${ }^{1}$ State Key Laboratory of Genetic Engineering, Institute of Biostatistics, School of Life Sciences, Fudan University, Shanghai, China ${ }^{2}$ Department of Information and Computing Science, School of Mathematics and Physics, Anhui University of Technology, Ma'anshan, China ${ }^{3}$ Shanghai Center for Mathematical Sciences, Fudan University, Shanghai, China ${ }^{4}$ Shanghai Public Health Clinical Center, Fudan University, Shanghai, China ${ }^{5}$ SJTU-Yale Joint Center for Biostatistics, Shanghai Jiao Tong University, Shanghai, China

Corresponding author:

Yue-Qing $\mathrm{Hu}^{1,3}$

Email address: yuehu@fudan.edu.cn

\begin{abstract}
Knowledge of similarities among diseases can contribute to uncovering common genetic mechanisms. Based on ranked gene lists, a couple of similarity measures were proposed in the literature. Notice that they may suffer from the determination of cutoff or heavy computational load, we propose a novel similarity score SimSIP among diseases based on gene ranks. Simulation studies under various scenarios demonstrate that SimSIP has better performance than existing rank-based similarity measures. Application of SimSIP in gene expression data of 18 cancer types from The Cancer Genome Atlas shows that $\operatorname{SimSIP}$ is superior in clarifying the genetic relationships among diseases and demonstrates the tendency to cluster the histologically or anatomically related cancers together, which is analogous to the pan-cancer studies. Moreover, SimSIP with simpler form and faster computation is more robust for higher levels of noise than existing methods and provides a basis for future studies on genetic relationships among diseases. In addition, a measure $M A G$ is developed to gauge the magnitude of association of an individual gene with diseases. By using $M A G$ the genes and biological processes significantly associated with colorectal cancer are detected.
\end{abstract}

\section{INTRODUCTION}

Exploring the common genetic basis of complex human diseases is often useful for understanding the disease relationships drawing on their genetic mechanisms. A similarity measure is a central component in detecting the common genetic basis among different diseases. Several common metrics have been proposed to measure similarities between diseases, such as Pearson, Spearman correlation coefficient, Euclidean distance, Manhattan distance, and Jaccard correlation coefficient (Antosh et al., 2013; Dennis et al., 2003; Serra et al., 2016; Shi et al., 2014). However, with the technological developments in molecular biology, large-scale gene expression profiling datasets produced from diverse technological platforms necessitate new and adaptive similarity measures to reveal meaningful genetic relationships across multiple platform types. Ranking genes according to their contribution to each disease can convert heterogeneous data into platform-independent rank lists, and a recent study (Serra et al., 2016) suggested that the discrimination ability of the similarity/distance measures based on ranked gene lists perform well or better than traditional measures (such as Euclidean distance and correlation coefficient), particularly for gene expression datasets produced with different biotechnologies (microarray, RNA-seq, etc.). Therefore, 
the genetic overlaps among diseases can be detected by integrating multi-platform datasets using similarity measures based on ranked gene lists, which can help us gain further insight on understanding disease etiology.

A review of related studies in similarity measures based on ranked gene lists showed that most of them depend on either a fixed cutoff position to consider overlaps between the top part of ranked gene lists or variational cutoffs to select the one that generates the most significant results (Chen et al., 2015; Dennis et al., 2003; Von Mering et al., 2006). To avoid the uncertainty of results owing to arbitrariness in cutoff settings, global rank-based similarity measures have been developed. For example, the algorithm of GOrilla is a tool to discover and visualize the enrichment of GO terms that ranks genes by fold-change (Eden et al., 2009). The algorithm of gene set enrichment analysis (GSEA) allows all genes to contribute to overlapping signals in proportion to their degree of differential expression and can detect the weak signals that would be discarded by 'threshold' approaches (Efron and Tibshirani, 2007; Subramanian et al., 2005). The algorithm of CORaL estimates the significant set size using the overlaps between sections of the ranked gene lists by maximizing statistical likelihood (Antosh et al., 2013). The algorithm of $R 2 K S$ particularly emphasizes finding the same items near the top of the ranked gene list (Ni and Vingron, 2012). Plaisier et al. (2010) presented a popular 'threshold-free' approach in neuroscience and biomedicine named rank-rank hypergeometric overlap $(R R H O)$, which identifies and visualizes regions of significant overlap between two ranked gene lists and determines the statistical significance of enrichment by hypergeometric distribution.

Moreover, a similarity measure OrderedList that focuses on evaluating whether there is significant overlap between two ranked gene lists was proposed (Yang et al., 2006). The OrderedList is the weighted sum of the number of overlapping genes with an exponentially decaying weight, where a parameter $\beta$ is introduced to determine how deep to go in the ranked gene lists. There are many studies on statistics-based improvement from similarity measures OrderedList. For example, Serra et al. (2016) introduced similarity measure $F E S_{\beta}$ (fraction enrichment sum) and set the exponentially decaying parameter $\beta$ as $0,0.001$, and 0.01. Chen et al. (2015) adopted the default series of $\beta$ values in the R package OrderedList and proposed a robust statistic minimum $p$ value: $\min _{\beta} p_{\beta}$, where parameter $\beta$ can be calculated by setting a minimum weight and a default series of positions. To avoid arbitrariness and manual intervention of parameter $\beta$ selection, Chen et al. (2015) finally found a parameter-free similarity measure WeiSumE* with good performance to detect overlaps among ranked gene lists in simulations, which is the weighted sum that normalizes the number of overlapping genes on the top genes of two ranked gene lists by its expectation. Generally, existing rank-based similarity measures are mostly based on the overlaps among ranked gene lists and may lead to information loss owing to fixed cutoff positions in the ranked gene lists, the uncertainty of results because of the arbitrariness of the cutoff position, or heavy computational burden.

In this study, we propose $M A G$ based on the transformation of gene ranks to measure the magnitude of association of the individual gene with two diseases. By exploring the summation of $M A G$ over all genes, we develop a novel similarity score SimSIP with simpler form and light computation burden to gauge genetic overlap among diseases based on gene ranks instead of intersections between the top part of ranked gene lists. To show the superiority of SimSIP, we firstly conduct a series of simulation studies to demonstrate the performance of SimSIP compared to some existing similarity measures based on ranked gene lists (WeiSumE* (Chen et al., 2015), OrderedList (Yang et al., 2006), FE $S_{0.001}, F E S_{0.01}$ (Serra et al., 2016)) and Euclidean distance $E u c D$ under various scenarios. Secondly, we apply SimSIP to analyze the gene expression data of cancers in The Cancer Genome Atlas database and find that it sheds light on the genetic relationships among cancers. Thirdly, we arrange the significantly similar cancer pairs among the 18 cancer types detected by SimSIP into a disease network in which the tendency to cluster the histologically or anatomically related cancers provides basic support for pan-cancer studies. Finally, for the most significantly similar cancer pair, colon adenocarcinoma $(C O A D)$ and rectum adenocarcinoma (READ), found by SimSIP, we use $M A G$ to measure the magnitude of association of each gene both with $C O A D$ and $R E A D$ and find the important oncogenes of colorectal cancer which are associated with $C O A D$ and $R E A D$ and regulated in the same pattern. Moreover, biological processes highly associated with colorectal cancer are detected. 
It is easily observed from the expression of SimSIP that the more identically ranked genes associated with diseases 1 and 2 there are, the more similar two diseases are, and the larger SimSIP is. Therefore, an observed SimSIP larger than expected under the null hypothesis that two diseases have non-overlapping genes means significant. When we say that two diseases have no overlapping genes in the genetic perspective, we mean that the two gene rank lists are two random shufflings of $1 \sim n$. Based on the expression of SimSIP, we describe a procedure to generate the empirical distribution of SimSIP under the null hypothesis, which is used to evaluate the significance of the similarity score. Without loss of generality, we fix the first gene rank list as $\{i\}_{i=1}^{n}$ and random permutate $1 \sim n$ as $\left\{b_{i}\right\}_{i=1}^{n}$ for $S$ times, then we obtain the corresponding $\operatorname{SimSIP}^{s}, s=1,2, \ldots, S$. The $p$ value of $\operatorname{SimSIP}$ is computed as

$$
p=\frac{\sum_{s=1}^{S} I\left(\operatorname{Sim} S I P^{s} \geq \operatorname{Sim} S I P\right)}{S},
$$

where $I(\cdot)$ is the indicator function assigning the value 1 or 0 relying on whether the condition within brackets is met. Let $p^{r}$ denote the $p$ value and $r=1,2, \ldots, R$ for $R$ replications, and the power under the 
alternative hypothesis or the type I error under the null hypothesis for a given significance level $\alpha$ is

$$
\text { Power }=\frac{\sum_{r=1}^{R} I\left(p^{r} \leq \alpha\right)}{R} .
$$

Regarding $M A G$, we can employ a similar procedure to evaluate the significance of an observed $M A G$. Under the null hypothesis that two diseases having non-overlapping genes, the two gene ranks $a_{i}$ and $b_{i}$ involved in are randomly drawn from $\{1,2, \ldots, n\}$ with replacement, and it is not difficult to get the distribution of $M A G$ under the null hypothesis. The significance of the observed $M A G$ can then be calculated.

\section{Results Simulation Study Parameter setting}

We carry out simulations to evaluate the performance of SimSIP and the existing rank-based methods

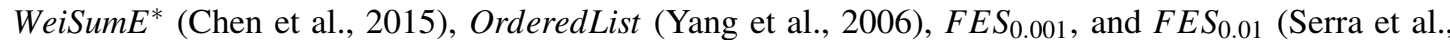
2016), which are weighted sum of the number of overlapping genes between the top part of ranked gene lists, and Euclidean distance $E u c D$ between the original values, which is a method with no need of ranking.

As done in Chen et al. (2015), we set the number of genes $n=6000,11000$, and 25000 and randomly choose two sets of $d$ genes from the $n$ genes as the associated genes with diseases 1 and 2, respectively. We fix the $d=1,5,10$, and 20 in the simulation. The number $o$ of overlapping associated genes in the two sets is taken as 0 for evaluating the type I error rate and positive for evaluating the power. For convenience, genes 1 to $d$ are assumed to be associated with disease 1 and genes $d-o+1$ to $2 d-o$ are associated with disease 2 , where $d \geq o$. As performed by Chen et al. (2015), two normal distributions $N\left(0, \sigma^{2}\right)$ and $N\left(1, \sigma^{2}\right)$ are employed to generate the rank of each gene. Specifically, $N\left(1, \sigma^{2}\right)$ is for the associated genes and $N\left(0, \sigma^{2}\right)$ for the remaining $n-d$ non-associated genes, in either disease 1 or 2 . The gene rank is from the value of normal distribution. The variance $\sigma^{2}$ in the normal distribution is taken as $0.01,0.05,0.1,0.5$ when $o=0 ; 0.07,0.09,0.11,0.13$ when $o=1 ; 0.1,0.15,0.2,0.25$ when $o=5 ; 0.2,0.25,0.3,0.35$ when $o=10$; and $0.3,0.35,0.4,0.45$ when $o=20$. The variance $\sigma^{2}$ plays an importance role in discerning the associated genes. The number of replications $R$ is set to 1000 in the computation of powers/type I error rates, and the nominal significance level is set to 0.05 . We set $S=1000$ in the simulation study and $S=10$ millions in real data analysis of TCGA. As an illustration, the empirical distribution of SimSIP for total genes number $n=6000,11000,25000$ are given in Figures S1-S3 and the empirical distribution of $M A G$ for $n=6000,11000,25000$ are given in Figures S4-S6.

\section{Type I error}

We firstly show the simulation results under the null model in evaluating the type I error rate of SimSIP and five existing methods under various scenarios. For the null setting, the empirical $p$ values of existing rank-based methods (WeiSumE ${ }^{*}$, OrderedList, $F E S_{0.001}, F E S_{0.01}$ ) are computed by permutating ranked gene lists from the value of the normal distribution, and the null distribution of $E u c D$ is obtained by permutating the value of the normal distribution generated. All empirical sizes shown in Supplementary Table S1 are around the significance 0.05 and are well controlled.

\section{Power comparison}

In the second set of simulations, the performance of SimSIP is compared with five other existing measures in terms of power. The results show that the powers of six measures are all monotonic decreasing for higher noise (variance $\sigma^{2}$ ) and monotonic increasing for a bigger ratio of $o$ to $d$. The findings are shown in Figure 1. Firstly, for the same total gene number $n$, the smaller the number of associated genes $d$ becomes, the more obvious the advantage of the SimSIP is. For example, in the scenarios of $d=1$ (in subfigures A, E and I of Figure 1), the power of SimSIP has almost a 10\% increase compared with that of WeiSumE $^{*}$ (Chen et al., 2015) and OrderedList (Yang et al., 2006), is more than 2 times the powers of $F E S_{0.01}$ (Serra et al., 2016), and almost more than 8 times the powers of $F E S_{0.001}$ (Serra et al., 2016) and $E u c D$. The scenario of $d=5$ is analogy to the scenario of $d=1$. However, in the scenarios of increasing $d$ up to 10 and 20, the advantage of SimSIP is no longer obvious. Secondly, for the same $d$, the larger the number of genes $(n)$ becomes, the more obvious the advantages of SimSIP is. For example, in the 


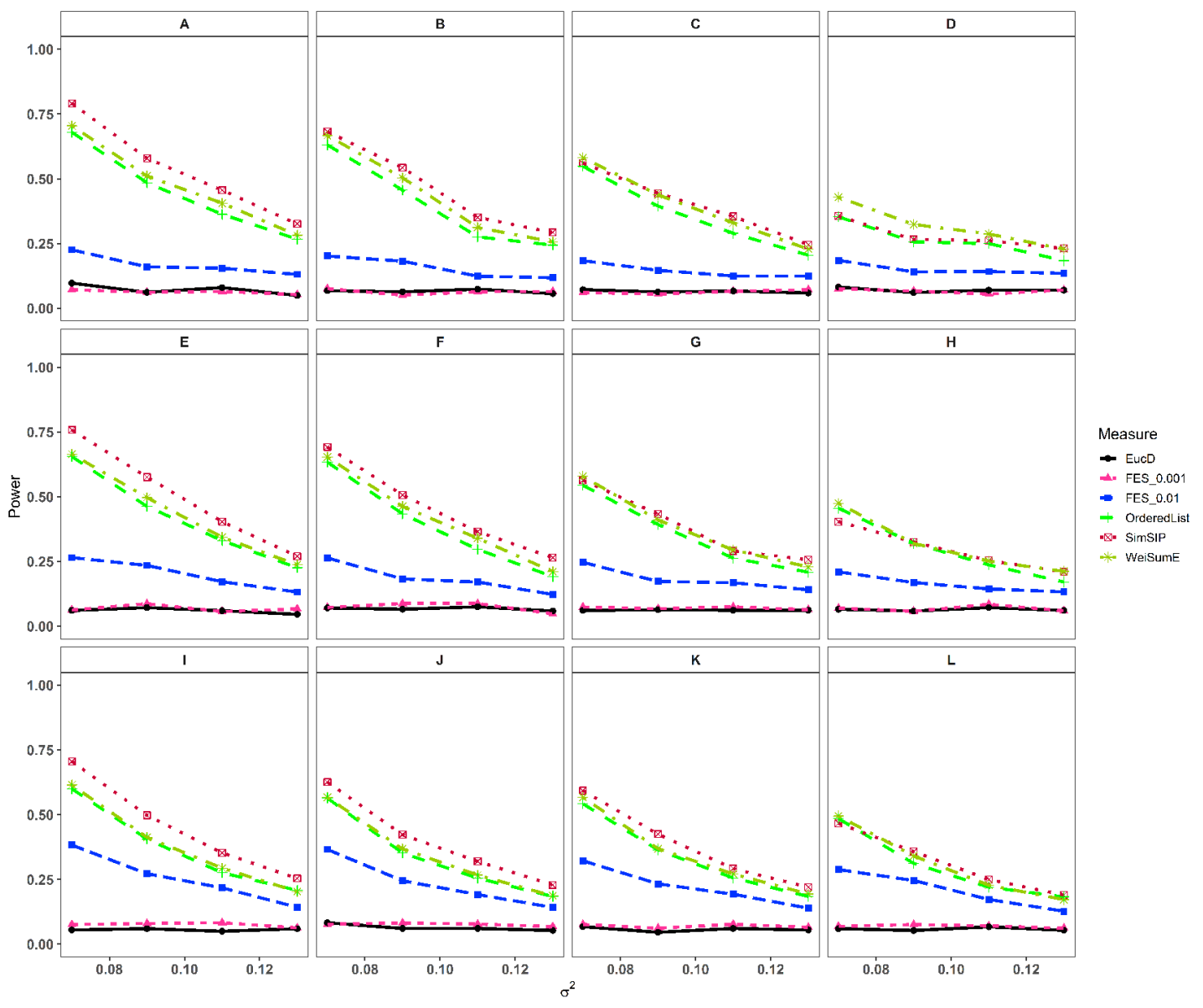

Figure 1. Powers of EucD, FES $S_{0.001}, F E S_{0.01}$, OrderedList, SimSIP and WeiSumE* when $o=1$ with 12 scenarios. (A) $\mathrm{n}=6000, \mathrm{~d}=1$; (B) $\mathrm{n}=6000, \mathrm{~d}=5$; (C) $\mathrm{n}=6000, \mathrm{~d}=10$; (D) $\mathrm{n}=6000, \mathrm{~d}=20$; (E) $\mathrm{n}=11000$, $\mathrm{d}=1$; (F) $\mathrm{n}=11000, \mathrm{~d}=5 ;$ (G) $\mathrm{n}=11000, \mathrm{~d}=10$; (H) $\mathrm{n}=11000, \mathrm{~d}=20$; (I) $\mathrm{n}=25000, \mathrm{~d}=1 ;$ (J) $\mathrm{n}=25000, \mathrm{~d}=5$; (K) $n=25000, d=10 ;(L) n=25000, d=20$. The arrangement of variance $\sigma^{2}$ on $x$ axis is a series $(0.07,0.09$, $0.11,0.13$ ) on which the power of six measures can be ranged from 0.1 to 0.95 .

scenarios of $n=6000$ and $n=11000$, the similarity measure WeiSumE ${ }^{*}$ is the most powerful when the number of associated genes $d=20$; however, SimSIP becomes superior when compared with the other five measures when $n$ increases to 25000. Thirdly, when the number of associated genes $d$ is relatively large, SimSIP gradually manifests certain advantages with the increase in noise (variance $\sigma^{2}$ ), which can be observed by six subfigures C, D, G, H, K and L in Figure 1 (in the scenarios of $d=10$ and $d=20$ ). When increasing the number of overlapping associated genes $o$ up to 5, SimSIP always maintains the superiority compared with the other five methods, and its power has obvious advantages for the larger ratio of $o$ to $d$ with a fixed $n$ and for the larger $n$ with a fixed $d$ (see Figure 2). When the number of overlapping associated genes $o$ increases from 5 to 10 or 20, the advantages of SimSIP are much more significant (see Figures S7-S8 in Supplementary Information).

As shown in Figure 1-2 and S7-S8, the similarity measure WeiSumE* performs better than other measures when there are less but significant overlap between two diseases with lower noise (variance $\sigma^{2}$ ), and our method SimSIP performs better in all the other scenarios. Especially for $n=25000$, which approximates the number of genes in the full human genome, the performance of SimSIP is always the best. Thus, compared with other measures, SimSIP is more appropriate for detecting genetic similarity between longer gene lists and works well when more overlapping genes occur among diseases. Most importantly, our method SimSIP is more robust for higher noise than the other five methods. Therefore, SimSIP is more suitable for the study of human diseases than the existing methods, especially for the study of cancers in which there are more genetic overlaps. 


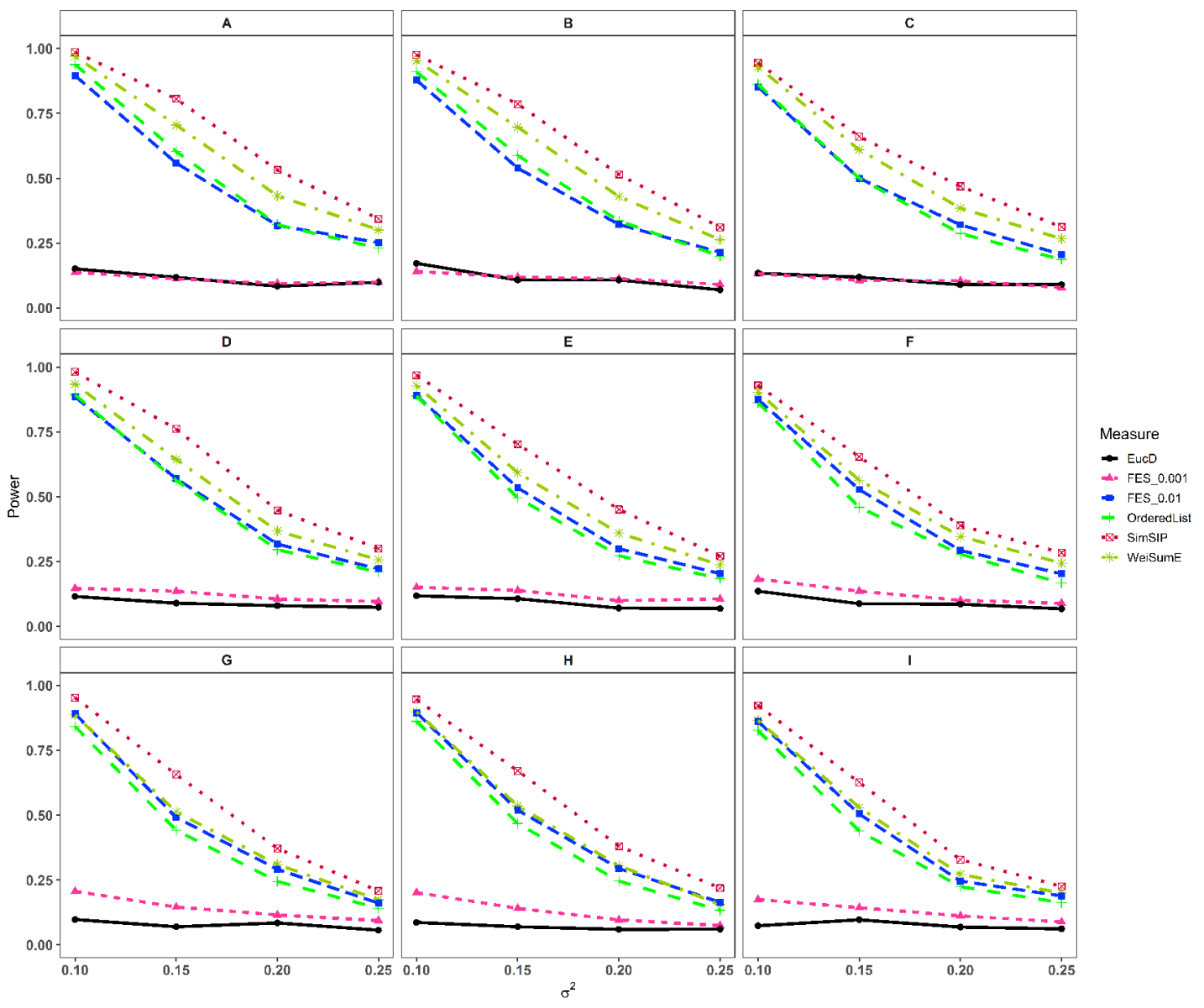

Figure 2. Powers of EucD, FES $S_{0.001}, F E S_{0.01}$, OrderedList, SimSIP and WeiSumE* when $o=5$ with 9 scenarios. (A) $\mathrm{n}=6000, \mathrm{~d}=5$; (B) $\mathrm{n}=6000, \mathrm{~d}=10$; (C) $\mathrm{n}=6000, \mathrm{~d}=20$; (D) $\mathrm{n}=11000, d=5$; (E) $\mathrm{n}=11000$, $\mathrm{d}=10 ;(\mathrm{F}) \mathrm{n}=11000, \mathrm{~d}=20 ;(\mathrm{G}) \mathrm{n}=25000, \mathrm{~d}=5 ;(\mathrm{H}) \mathrm{n}=25000, \mathrm{~d}=10 ;(\mathrm{I}) \mathrm{n}=25000, \mathrm{~d}=20$. The arrangement of variance $\sigma^{2}$ on $x$ axis is a series $(0.1,0.15,0.2,0.25)$ on which the power of six measures can be ranged from 0.1 to 0.95 .

Furthermore, the similarity measure OrderedList has a higher power with fewer overlaps (for $o=1$ ) and $F E S_{0.01}$ has a higher power with more overlaps (for $o>1$ ). Their performances are superior to those of similarity measure $F E S_{0.001}$ and Euclidean distance $E u c D$, which always have low power in all scenarios. We also find that the rank-based measures always perform better than Euclidean distance EucD, which uses the value of the normal distribution rather than gene ranks. These results demonstrate that the rank of a gene can provide additional information on its contribution to each disease upon converting real data to ranks.

In general, compared with existing similarity measures, SimSIP performs better in almost every simulation, which indicates that it is feasible to construct similarity measure by replacing overlaps between top ranked gene sets with gene ranks.

\section{TCGA Data Analysis}

TCGA is a combined effort by multiple research institutes, in which tumor and normal samples from more than 11000 patients are publicly available, comprising 37 types of (epi)genetic and clinical data for 33 types of cancer. We download gene expression datasets (whose gene expression profiles were determined experimentally using the Illumina HiSeq 2000 RNA Sequencing platform) of 33 types of cancer using the UCSC Xena functional genome browser (https: //xenabrowser.net/datapages/). Expression data of 20530 genes are available for each cancer, and 18 types of cancer are selected (see details in Table 1) based on the criteria that the sample size of the control group is not smaller than 5 . We rank all genes 
Table 1. Sample sizes of control group and case group per cancer type in TCGA

\begin{tabular}{llll}
\hline Abbreviation & Cancer type $^{a}$ & $n_{0}{ }^{b}$ & $n_{1}$ \\
\hline BLCA & Bladder urothelial carcinoma & 19 & 407 \\
BRCA & Breast invasive carcinoma & 114 & 1097 \\
CHOL & Cholangiocarcinoma & 9 & 36 \\
COAD & Colon adenocarcinoma & 41 & 286 \\
ESCA & Esophageal carcinoma & 11 & 184 \\
GBM & Glioblastoma multiforme & 5 & 154 \\
HNSC & Head and neck squamous cell carcinoma & 44 & 520 \\
KICH & Kidney chromophobe & 25 & 66 \\
KIRC & Kidney renal clear cell carcinoma & 72 & 533 \\
KIRP & Kidney renal papillary cell carcinom & 32 & 290 \\
LIHC & Liver hepatocellular carcinoma & 50 & 371 \\
LUAD & Lung adenocarcinoma & 59 & 515 \\
LUSC & Lung squamous cell carcinoma & 51 & 502 \\
PRAD & Prostate adenocarcinoma & 52 & 497 \\
READ & Rectum adenocarcinoma & 10 & 94 \\
STAD & Stomach adenocarcinoma & 35 & 415 \\
THCA & Thyroid carcinoma & 59 & 505 \\
UCEC & Uterine corpus endometrial carcinoma & 24 & 176 \\
\hline${ }^{a}$ From 33 types of cancer in TCGA, 18 types of cancer with control group sample size being 5 or \\
more are selected. \\
b $n_{0}$ is sample size of control group and $n_{1}$ is sample size of case group for a given cancer
\end{tabular}

by their $p$ values derived from the R package LIMMA for differential expression analysis and apply our proposed method to gauge the genetic similarity among the 18 types of cancer in TCGA. In this section, we choose WeiSumE* (Chen et al., 2015), which performs only second to SimSIP under all simulation scenarios, to compare with SimSIP.

For the $(18 \times 17) / 2=153$ cancer pairs among 18 cancers, we compute their similarity scores by using SimSIP and WeiSumE* respectively and standardize them by normalization method $\frac{T-T_{\min }}{T_{\max }-T_{\min }}$. The normalized similarity scores about SimSIP are in [0.11, 0.77] with mean 0.24 and variance 0.015 , and that about $W_{e i S u m E}^{*}$ are in $[0.064,0.53]$ with mean 0.13 and variance 0.005 . Obviously, the normalized similarity scores about SimSIP are generally bigger and more spread than those about WeiSumE* ${ }^{*}$. So SimSIP is more discriminative than WeiSumE* in quantifying the relationships among diseases.

\section{Significant cancer pairs in TCGA}

To illustrate the application of SimSIP and WeiSumE* in exploring significant relationships among 18 cancers, we compute the empirical $p$ values of SimSIP and WeiSumE* based on the null distribution (shown in Figures S9-S10) for the 153 cancer pairs among 18 cancers.

Given the nominal significance level of 0.05 , the Bonferroni adjustment of empirical $p$ values $0.05 / 153$ is employed to detect significant similar cancer pairs. 91 significant similar cancer pairs are detected by SimSIP 82 pairs by WeiSumE*, with 81 pairs in their intersection (see Table S2 and Figure S11). For the 81 cancer pairs detected both with SimSIP and WeiSumE* (Chen et al., 2015), Figure 3 displays the empirical $p$ values of the two methods for the same cancer pair. Clearly, the empirical $p$ values of SimSIP are generally smaller than that of WeiSumE* (except for cancer pair KIRP and $L U A D$ with index 63 in Figure 3), which is consistent with the results given in the simulation.

As shown in Figure S11, SimSIP finds more possibly significant cancer pairs which include almost all the significant cancer pairs found with WeiSumE* ${ }^{*}$. Among the 10 significant cancer pairs (shown in red in Table S2) found with SimSIP, not WeiSumE*, five cancer pairs can be explained in pan-cancer studies: For cancer pair $C O A D$ and $U C E C$, the diversity of high antigen-specific TCR repertoires correlates with the improved prognostic progression-free interval in COAD and UCEC (Thorsson et al., 2018); the expression of TMEM173 in tumor tissues is significantly upregulated and hypomethylated in cancer pair $C O A D$ and THCA but significantly downregulated and hypermethylated in cancer pair $L U S C$ and PRAD (An 
et al., 2019). Furthermore, there are high mutations rates for TBK1 in cancer pair COAD and UCEC, and the expression of TMEM173 is positively associated with the infiltration of immune cells in cancer pair BRCA and THCA (An et al., 2019). A high IRF3 expression yields a poor prediction of prognosis in patients with KIRC and PRAD (An et al., 2019). In addition, cancer pair COAD and UCEC depends on components of the EGFR pathway at similar frequencies (Wormald et al., 2013).

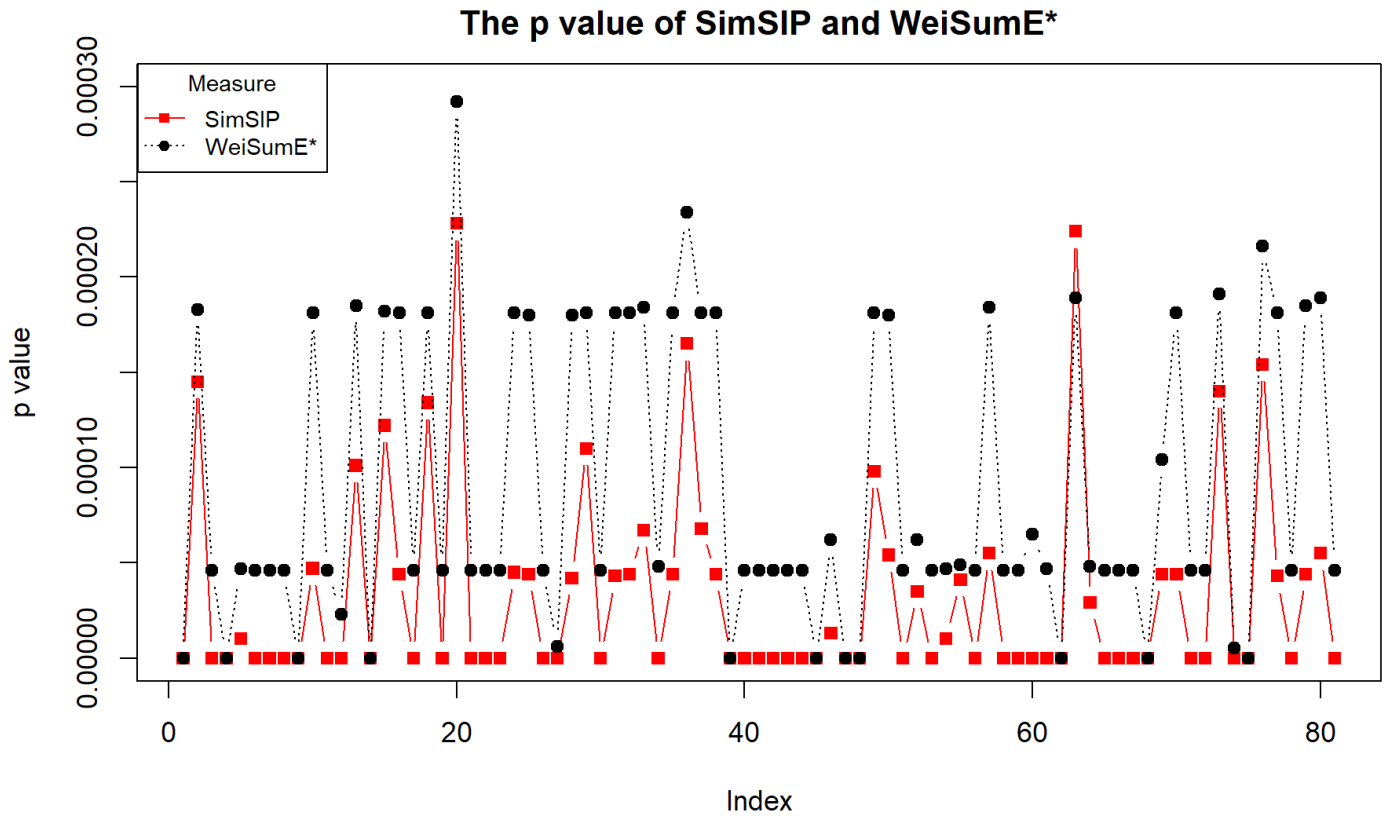

Figure 3. The empirical $p$ values of SimSIP (black dot) and WeiSumE* (red square) for 81 cancer pairs found with both SimSIP and WeiSumE*.

\section{Genetic similarity among 18 types of cancer}

Depicting the 91 significant similar cancer pairs found with SimSIP in a disease network by using software Cytoscape (https : //cytoscape.org/) as in Figure 4, the following observations could be addressed:

1. The vertex HNSC has the largest degree, 15, which shows that the HNSC has significant similarity with the other 15 cancers, except KICH and KIRC. The degrees of vertices such as BLCA and UCEC reach 14; READ, ESCA, LUSC, and HNSC reach 13; and LUAD and $L I H C$ reach 12. There are close intrinsic genetic relationships among the 18 cancers. The seven types of cancers ESCA, LUSC, HNSC, BLCA, BRCA, STAD, and UCEC (their vertices with deeper color and bigger size) with a higher degree are closer to each other and tend to form a pivotal hub of the disease network of 18 cancer types.

2. Cancers originating from the same organ or tissue tend to co-cluster, such as cancer pair READ and COAD or KIRP and KIPC with the most similar relationship. In addition, cancers with proximity also tend to group together, such as LIHC and CHOL with a highly significant relationship. These provide evidence that tumors with closer physical distance in human organs have similar sources of endoderm development or exposure to a common cancer-causing environmental factor (Sell and Dunsford, 1989). Further, for the three types of kidney tumors, KICH, KIRP, and KIPC, the similarity between KIRP and KIPC is more significant than KICH and KIPC or KIRP and KIPC, which may be explained by the fact that KIRC and KIRP are cancers of the proximal tubule segments, whereas KICH is a cancer of the distal tubule segments (Chen et al., 2016; Davis et al., 2014; Lee et al., 2015). Compared to other cancer pairs from the same tissue, the degree of similarity between the two types of lung tumor LUSC and LUAD is not so significant, which may be due to the derivation of cell types of the two types of lung tumors: LUSC originates from squamous epithelial cells in the respiratory tract and alveoli, whereas LUAD originates from a large number of glandular or alveolar cells (Li et al., 2015; Mainardi et al., 2014; Sutherland et al., 2014). 


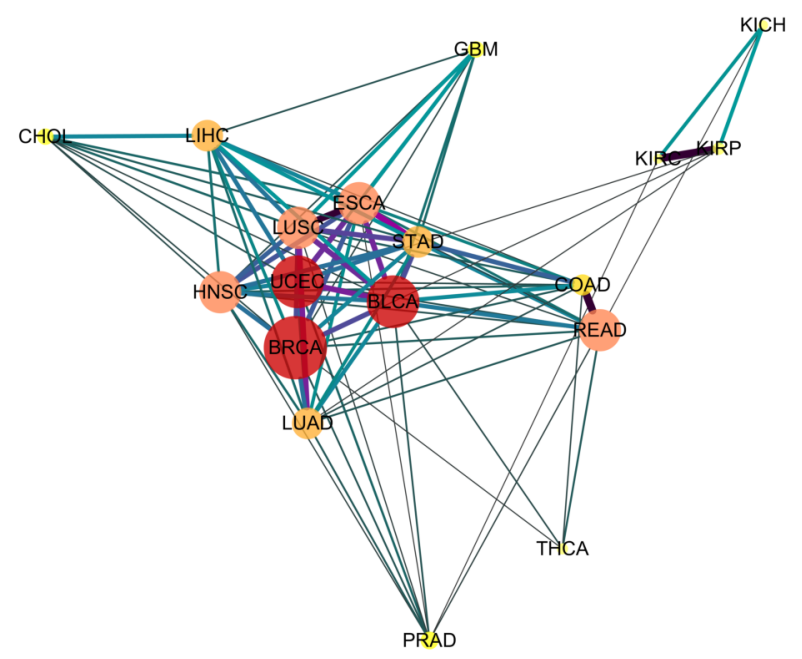

Figure 4. The diseases network of 18 cancer types. Taking Bonferroni adjustment of empirical $p$ values given nominal significance 0.05 as a threshold, we select 91 significantly similar cancer pairs found with SimSIP to demonstrate the genetic relationships among cancers by software Cytoscape

(https: //cytoscape.org/). The width, length and color of the edge in the network are determined by the magnitude of SimSIP, the size and color of the vertex in the network is determined by the degree of vertex (the number of cancers which is significantly related to this cancer).

3. There are significant similarities among gastrointestinal tumors (READ, COAD, STAD, and ESCA), which is consistent with the results of integrative clustering across data types in the miRNA, mRNA, and RPPA platforms (Hoadley et al., 2018). In the disease network (Figure 4), squamous cell carcinomas (BLCA, ESCA, HNSC, and LUSC) are also co-clustered, and the similarity score of the cancer pair ESCA and LUSC is the top three and has more significant similarity. Hoadley et al. (2018) reported a similar discovery based on a multi-platform dataset (including miRNA, mRNA, RPPA, aneuploidy, and DNA methylation data) in TCGA, and Abrams et al. (2018) also suggested that regardless of the tissue types of squamous cell carcinoma, potential similarities were detected among the transcription factor expression profiles of BLCA, ESCA, HNSC, and LUSC. In addition, the gynecologic tumors UCEC and BRCA are also close to each other in the network, which is consistent with the results of previous studies (Hoadley et al., 2018).

4. Finally, similar to previous studies (Abrams et al., 2018; Hoadley et al., 2018), three types of cancer, PRAD, THCA, and GBM, are relatively independent in the network, the relationships among them and other cancers are relatively weak. In addition, instead of squamous cancers being clustered together, adenocarcinomas (PRAD, COAD, LUAD, STAD, and READ) appear to be scattered around the edge of the network.

From the above observations of the disease network (Figure 4) obtained with SimSIP, we find them to be analogous to those of previous pan-cancer studies (Abrams et al., 2018; Hoadley et al., 2018): histologically or anatomically related cancers tend to cluster together, which provide basic support for analyses of pan-gastrointestinal, pan-squamous, pan-kidney, and pan-gynecological cancers.

\section{Associated genes with colorectal cancer}

For the most significantly similar cancer pair COAD and READ found with SimSIP, we try to explore the common underlying genetic mechanisms by sorting $M A G$ values of genes in descending order. The significance of the difference of expression level of the top 5 genes in $C O A D$ and $R E A D$ are shown in Table S3, which are obtained from the TCGA data mining website UALCAN (http://ualcan.path.uab.edu/). Table S3 provides evidence that the top 5 genes are associated with COAD and READ and they are regulated in the same pattern (either up-regulated or down-regulated). It is worth mentioning that, for the top 500 genes associated with $C O A D$ and $R E A D$ and the top 500 genes associated with $K I C H$ and $S T A D$, which are the most similar and the least similar cancer pairs found with SimSIP respectively, the proportion of genes regulated in the same pattern is 1 and 0.648 respectively. Furthermore, we compute 
Table 2. The result of pathway enrichment analysis of colorectal cancer

\begin{tabular}{lll}
\hline ID & KEGG pathway & Benjamini \\
\hline hsa04713 & Circadian entrainment & $2.30 \mathrm{E}-04$ \\
hsa04024 & cAMP signaling pathway & $2.97 \mathrm{E}-04$ \\
hsa03008 & Ribosome biogenesis in eukaryotes & $3.68 \mathrm{E}-04$ \\
hsa04080 & Neuroactive ligand-receptor interaction & $7.92 \mathrm{E}-04$ \\
hsa04020 & Calcium signaling pathway & 0.003328211 \\
hsa04723 & Retrograde endocannabinoid signaling & 0.006342379 \\
hsa04724 & Glutamatergic synapse & 0.006620968 \\
hsa04911 & Insulin secretion & 0.010800326 \\
hsa04728 & Dopaminergic synapse & 0.011092804 \\
hsa04725 & Cholinergic synapse & 0.017964832 \\
hsa04022 & cGMP-PKG signaling pathway & 0.021342644 \\
hsa04970 & Salivary secretion & 0.023476401 \\
hsa04261 & Adrenergic signaling in cardiomyocytes & 0.024338123 \\
hsa00071 & Fatty acid degradation & 0.029045641 \\
hsa05032 & Morphine addiction & 0.036794268 \\
hsa04978 & Mineral absorption & 0.038043761 \\
hsa04971 & Gastric acid secretion & 0.045576 \\
hsa04726 & Serotonergic synapse & 0.045974199 \\
hsa00910 & Nitrogen metabolism & 0.047420936 \\
hsa05033 & Nicotine addiction & 0.047658725 \\
hsa05031 & Amphetamine addiction & 0.048006097 \\
\hline
\end{tabular}

\section{Associated signaling pathway with colorectal cancer} gories.

the correlation coefficient between the logarithmic transformation of SimSIP and the proportion of genes regulated in the same pattern in the top $N(N=500,1000,1500,2000)$ genes associated with each of 153 cancer pairs (shown in Figure S12). Obviously, there is a significant relationship between the degree of overlap between diseases and the proportion of genes regulated in the same pattern. These findings suggest that the associated genes of highly overlapping cancers may be regulated in the same pattern.

Through gene annotation by using Metascape (http://metascape.org/) and literature review, among the top 5 genes, one gene (CDH3) is associated with multiple cancers (shown in Figure S13), and two genes (ETV4, KRT24) are associated with colorectal cancer; the remaining genes can be considered as candidate susceptibility genes of colorectal cancer (Table S4). CDH3, the top 1 gene detected by MAG, is located in a region on the long arm of chromosome 16. Paredes et al. (2012) advised that genetic or epigenetic changes in this gene or changes in its protein expression often lead to tissue disorders, cellular dedifferentiation, and enhanced invasiveness of tumor cells. This gene is associated with intestinal infections (Van Marck et al., 2011) and colon cancer (Van Marck et al., 2011; Sun et al., 2011). In addition, its over-expression is also associated with tumor progression and low survival in non-small-cell lung cancer (Imai et al., 2018) and in the loss of heterozygous events for breast and prostate cancer (Royo et al., 2016; Sousa et al., 2014; Vieira et al., 2017). Moreover, CDH3 is significantly associated with liver cancer, gastric cancer, bladder cancer, and cervical adenocarcinoma (Bauer et al., 2014; Paredes et al., 2012; Sun et al., 2011; Van Marck et al., 2011). Searching for CDH3 in the CancerMine database (https://www.mycancergenome.org/) reveals that 12 cancers are associated with the over-expression of $\mathrm{CDH} 3$. This gene is therefore very important in the genetic mechanism of cancer. The top 3 gene ETV4 is strongly linked to metastasis of colorectal cancer (Dumortier et al., 2018) and enriched in pathway transcriptional misregulation in cancer (Table S4). The top 5 gene KRT24 is over-expressed in patients with colorectal cancer and is a susceptibility gene for early onset of colorectal cancer (Hong et al., 2007).

Calculating empirical $p$ values of $M A G$ for each gene by its null distribution (described in Figure S14), 1838 genes significantly associated with colorectal cancer are obtained. By DAVID (https://david.ncifcrf.gov /tools.jsp), these 1838 genes associated with colorectal cancer are clustered in diverse functional cate- 
As shown in Table 2, of 21 functional categories, 3 are associated with cancer (cAMP signaling pathway (Akgul, 2009; Myklebust et al., 1999), fatty acid degradation (Currie et al., 2013), and nitrogen metabolism (Sanchez-Vega et al., 2018)) and 11 appear in colorectal cancer studies, including calcium signaling pathway (Dallol et al., 2003), circadian entrainment (Lévi et al., 2010), ribosome biogenesis in eukaryotes (Lafontaine, 2015), cGMP-PKG signaling pathway (Li et al., 2013), dopaminergic synapse (Xu et al., 2010), retrograde endocannabinoid signaling (Proto et al., 2012), cholinergic synapse (Frucht et al., 1999), insulin secretion (Giovannucci, 2001), gastric acid secretion (Morton et al., 2011), morphine addiction (Jin et al., 2012), and nicotine addiction (Shureiqi et al., 2003; Yang and Frucht, 2001). The remaining signaling pathways can be considered as candidates for studies on biological processes associated with colorectal cancer.

In detail, the circadian entrainment pathway closely interacts with the cell division cycle and pharmacological pathways in the treatment of metastatic colorectal cancer and accelerates or slows down cancer growth through modifications of host and tumor circadian clocks, which drives $24 \mathrm{~h}$ changes in drug metabolism, cellular proliferation and apoptosis, cell cycle events, DNA repair, and angiogenesis (Lévi et al., 2007, 2010). For the insulin secretion pathway, because insulin and insulin-like growth factor axes are major determinants of cell proliferation and apoptosis, an increase in their circulating concentrations is associated with a high risk of colonic neoplasia. However, the dietary pattern with high saturated fatty acid intake can stimulate insulin resistance or secretion (Giovannucci, 2001), and cellular proliferation requires fatty acids to synthesize cell membranes and signaling molecules (Currie et al., 2013). In addition, the growth of colon tumor cells is selectively inhibited by nonsteroidal anti-inflammatory drugs that activate the cGMP/PKG pathway to suppress $\mathrm{Wnt} / \beta$-catenin signaling (Li et al., 2013). Up to $15 \%$ of colorectal cancers are distinguished by DNA microsatellite instability and manifested by the presence of DNA replication errors (Jass et al., 1998).

\section{DISCUSSION}

SimSIP is a novel similarity score that measures the genetic relationships among diseases by (1) introducing a suitable transformation of gene ranks that converts gene ranks into the magnitude of association of gene with the disease; and (2) comparing the similarity between gene rank lists in geometric space instead of comparing the overlaps between the top part of ranked gene lists. In this study, three additional tasks are also fulfilled: constructing a disease network of 18 cancers and offering some support for pan-cancer studies; developing a measure $M A G$ to gauge the magnitude of association of an individual gene with two diseases (note that $M A G$ can be generalized to multiple diseases); and finding some important genes and signaling pathways associated with colorectal cancer.

Extensive simulations show that SimSIP has better performance than existing methods in scenarios with larger numbers of overlapping associated genes $(o)$ and larger number of genes $(n)$, whereas in scenarios with smaller $o$ and smaller $n$, such as $o=1, n=6000$ or 11000 , WeiSumE* performs better. It is desirable that SimSIP can clearly identify the differences among diseases with more overlapping associated genes. Thus, in real data application, we use SimSIP to measure genetic similarities among cancers based on the differential expression analyses of multiple datasets in TCGA with the R package LIMMA. The results show that SimSIP can find more significant cancer pairs than WeiSumE ${ }^{*}$, such as cancer pair COAD and UCEC, COAD and THCA, LUSC and PRAD, BRCA and THCA, or KIRC and $P R A D$ demonstrating the usefulness of SimSIP. Furthermore, for the 81 cancer pairs found with both SimSIP and WeiSumE* , the $p$ value of SimSIP is smaller than WeiSumE* therefore, the SimSIP may be more powerful than WeiSumE* in detecting the significant cancer pairs.

Overall, SimSIP has a simpler form and faster computation (time consumption for six measures is shown in Supplementary Table S5), is more robust for higher levels of noise, and is more suitable for the study of human diseases than existing methods, especially for the study of cancers in which there are more genetic overlaps. In order to make this conclusion considerably stronger, we extend the range of the simulations to cover bigger number of overlapping associated genes, such as $o=50,100$ and 200 . As shown in Supplementary Figure S15-S17, SimSIP still performs well or better in the scenarios with bigger ratio of $o$ to $d$ when $o=50,100$ and 200, which is analogous to previous simulations. 


\section{CONCLUSIONS}

This article proposes a similarity score based on lists of gene ranks to measure the genetic relationships among diseases from gene expression data. Our method SimSIP gives a new perspective to detect the genetic relationships among diseases that does not depend on a threshold as fraction enrichment (Serra et al., 2016) nor on the weighted sum of overlaps between ranked gene lists as OrderedList (Yang et al., 2006).

Similar to other rank-based measures, our method relies on the correctness and scientific quality of the gene ranking. If gene ranking does not reflect the contribution of the gene to each disease, the rank-based measure is not necessarily superior to the commonly used measures. There are many common methods for ranking genes in practice, such as by the magnitude of $p$ values of t-test, of marginal regression analysis and of some methods for detecting differentially expressed genes (say LIMMA, edgeR and DESeq 2 et al.). Although $p$ values derived from the R package LIMMA for differential expression analysis were used in this paper to rank the gene, different researchers can choose different ranking methods based on their specific needs. In summary, in contrast to existing measures that are all based on the number of overlapping genes in top ranked gene lists among diseases, we creatively describe the genetic relationships among diseases from the spatial similarity between the transformation of gene ranks, which provides a new research direction for studies of similarity measures to reveal genetic relationships among diseases.

\section{ACKNOWLEDGMENTS}

We thank two anonymous reviewers for their constructive comments and suggestions that improve the presentation of the manuscript greatly. we thank our lab partners Liming Li and Yuquan Wang for code guidance, and our colleagues Zhongzhi Wang, Yongjin Zhang, and Wenxi Li for career help.

\section{REFERENCES}

Abrams, Z. B., Zucker, M., Wang, M., Taheri, A. A., Abruzzo, L. V., and Coombes, K. R. (2018). Thirty biologically interpretable clusters of transcription factors distinguish cancer type. BMC Genomics, 19(1):738.

Akgul, C. (2009). Mcl-1 is a potential therapeutic target in multiple types of cancer. Cellular and Molecular Life Sciences, 66(8):1326-1336.

An, X., Zhu, Y., Zheng, T., Wang, G., Zhang, M., Li, J., Ji, H., Li, S., Yang, S., Xu, D., Li, Z., Wang, T., He, Y., Zhang, L., Yang, W., Zhao, R., Hao, D., and Li, X. (2019). An analysis of the expression and association with immune cell infiltration of the cGAS/STING pathway in pan-cancer. Molecular Therapy-Nucleic Acids, 14:80-89.

Antosh, M., Fox, D., Cooper, L. N., and Neretti, N. (2013). CORaL: comparison of ranked lists for analysis of gene expression data. Journal of Computational Biology, 20(6):433-443.

Bauer, R., Valletta, D., Bauer, K., Thasler, W. E., Hartmann, A., Müller, M., Reichert, T. E., and Hellerbrand, C. (2014). Downregulation of P-cadherin expression in hepatocellular carcinoma induces tumorigenicity. International Journal of Clinical and Experimental Pathology, 7(9):6125.

Chen, F., Zhang, Y., Şenbabaoğlu, Y., Ciriello, G., Yang, L., Reznik, E., Shuch, B., Micevic, G., De Velasco, G., Shinbrot, E., Noble, M. S., Lu, Y., Covington, K. R., Xi, L., Drummond, J. A., Muzny, D., Kang, H., Lee, J., Tamboli, P., Reuter, V., Shelley, C. S., Kaipparettu, B. A., Bottaro, D. P., Godwin, A. K., Gibbs, R. A., Getz, G., Kucherlapati, R., Park, P. J., Sander, C., Henske, E. P., Zhou, J. H., Kwiatkowski, D. J., Ho, T. H., Choueiri, T. K., Hsieh, J. J., Akbani, R., Mills, G. B., Hakimi, A. A., Wheeler, D. A., and Creighton, C. J. (2016). Multilevel genomics-based taxonomy of renal cell carcinoma. Cell Reports, 14(10):2476-2489.

Chen, Q., Zhou, X. J., and Sun, F. (2015). Finding genetic overlaps among diseases based on ranked gene lists. Journal of Computational Biology, 22(2):111-123.

Currie, E., Schulze, A., Zechner, R., Walther, T. C., and Farese Jr, R. V. (2013). Cellular fatty acid metabolism and cancer. Cell Metabolism, 18(2):153-161.

Dallol, A., Morton, D., Maher, E. R., and Latif, F. (2003). SLIT2 axon guidance molecule is frequently inactivated in colorectal cancer and suppresses growth of colorectal carcinoma cells. Cancer Research, 63(5):1054-1058.

Davis, C. F., Ricketts, C. J., Wang, M., Yang, L., Cherniack, A. D., Shen, H., Buhay, C., Kang, H., Kim, S. C., Fahey, C. C., Hacker, K. E., Bhanot, G., Gordenin, D. A., Chu, A., Gunaratne, P. H., Biehl, M., 
Seth, S., Kaipparettu, B. A., Bristow, C. A., Donehower, L. A., Wallen, E. M., Smith, A. B., Tickoo, S. K., Tamboli, P., Reuter, V., Schmidt, L. S., Hsieh, J. J., Choueiri, T. K., Hakimi, A. A., The Cancer Genome Atlas Research Network, Chin, L., Meyerson, M., Kucherlapati, R., Park, W.-Y., Robertson, A. G., Laird, P. W., Henske, E. P., Kwiatkowski, D. J., Park, P. J., Morgan, M., Shuch, B., Muzny, D., Wheeler, D. A., Linehan, W. M., Gibbs, R. A., Rathmell, W. K., and Creighton, C. J. (2014). The somatic genomic landscape of chromophobe renal cell carcinoma. Cancer Cell, 26(3):319-330.

Dennis, G., Sherman, B. T., Hosack, D. A., Yang, J., Gao, W., Lane, H. C., and Lempicki, R. A. (2003) DAVID: database for annotation, visualization, and integrated discovery. Genome Biology, 4(9):R60.

Dumortier, M., Ladam, F., Damour, I., Vacher, S., Bièche, I., Marchand, N., de Launoit, Y., Tulasne, D., and Chotteau-Lelièvre, A. (2018). ETV4 transcription factor and MMP13 metalloprotease are interplaying actors of breast tumorigenesis. Breast Cancer Research, 20(1):73.

Eden, E., Navon, R., Steinfeld, I., Lipson, D., and Yakhini, Z. (2009). GOrilla: a tool for discovery and visualization of enriched GO terms in ranked gene lists. BMC Bioinformatics, 10(1):48.

Efron, B. and Tibshirani, R. (2007). On testing the significance of sets of genes. The Annals of Applied Statistics, 1(1):107-129.

Frucht, H., Jensen, R. T., Dexter, D., Yang, W.-L., and Xiao, Y. (1999). Human colon cancer cell proliferation mediated by the M3 muscarinic cholinergic receptor. Clinical Cancer Research, 5(9):25322539.

Giovannucci, E. (2001). Insulin, insulin-like growth factors and colon cancer: a review of the evidence. The Journal of Nutrition, 131(11):3109S-3120S.

Hoadley, K. A., Yau, C., Hinoue, T., Wolf, D. M., Lazar, A. J., Drill, E., Shen, R., Taylor, A. M., Cherniack, A. D., Thorsson, V., Akbani, R., Bowlby, R., Wong, C. K., Wiznerowicz, M., Sanchez-Vega, F., Robertson, A. G., Schneider, B. G., Lawrence, M. S., Noushmehr, H., Malta, T. M., The Cancer Genome Atlas Network, S., M, J., Benz, C. C., and Laird, P. W. (2018). Cell-of-origin patterns dominate the molecular classification of 10,000 tumors from 33 types of cancer. Cell, 173(2):291-304.

Hong, Y., Ho, K. S., Eu, K. W., and Cheah, P. Y. (2007). A susceptibility gene set for early onset colorectal cancer that integrates diverse signaling pathways: implication for tumorigenesis. Clinical Cancer Research, 13(4):1107-1114.

Imai, S., Kobayashi, M., Takasaki, C., Ishibashi, H., and Okubo, K. (2018). High expression of P-cadherin is significantly associated with poor prognosis in patients with non-small-cell lung cancer. Lung Cancer, 118:13-19.

Jass, J., Do, K.-A., Simms, L., Iino, H., Wynter, C., Pillay, S., Searle, J., Radford-Smith, G., Young, J., and Leggett, B. (1998). Morphology of sporadic colorectal cancer with DNA replication errors. Gut, 42(5):673-679.

Jin, S., Wang, S., Gao, X., and Xie, G. (2012). Effects of postoperative analgesia with dezocine and morphine on T-lymphocyte subsets and NK cell in colorectal cancer patients. Acta Academiae Medicinae Qingdao Universitatis, 48(4):364-370.

Lafontaine, D. L. (2015). Noncoding RNAs in eukaryotic ribosome biogenesis and function. Nature Structural \& Molecular Biology, 22(1):11.

Lee, J. W., Chou, C.-L., and Knepper, M. A. (2015). Deep sequencing in microdissected renal tubules identifies nephron segment-specific transcriptomes. Journal of the American Society of Nephrology, 26(11):2669-2677.

Lévi, F., Focan, C., Karaboué, A., de la Valette, V., Focan-Henrard, D., Baron, B., Kreutz, F., and Giacchetti, S. (2007). Implications of circadian clocks for the rhythmic delivery of cancer therapeutics. Advanced Drug Delivery Reviews, 59(9-10):1015-1035.

Lévi, F., Okyar, A., Dulong, S., Innominato, P. F., and Clairambault, J. (2010). Circadian timing in cancer treatments. Annual Review of Pharmacology and Toxicology, 50:377-421.

Li, F., He, J., Wei, J., Cho, W. C., and Liu, X. (2015). Diversity of epithelial stem cell types in adult lung. Stem Cells International, 2015:1-11.

Li, N., Xi, Y., Tinsley, H. N., Gurpinar, E., Gary, B. D., Zhu, B., Li, Y., Chen, X., Keeton, A. B., Abadi, A. H., Moyer, M. P., Grizzle, W. E., Chang, W.-C., Clapper, M. L., and Piazza, G. A. (2013). Sulindac selectively inhibits colon tumor cell growth by activating the cGMP/PKG pathway to suppress wnt/ $\beta$-catenin signaling. Molecular Cancer Therapeutics, 12(9):1848-1859.

Mainardi, S., Mijimolle, N., Francoz, S., Vicente-Dueñas, C., Sánchez-García, I., and Barbacid, M. (2014). Identification of cancer initiating cells in K-Ras driven lung adenocarcinoma. Proceedings of the 
National Academy of Sciences, 111(1):255-260.

Morton, M., Prendergast, C., and Barrett, T. D. (2011). Targeting gastrin for the treatment of gastric acid related disorders and pancreatic cancer. Trends in Pharmacological Sciences, 32(4):201-205.

Myklebust, J. H., Josefsen, D., Blomhoff, H. K., Levy, F. O., Naderi, S., Reed, J. C., and Smeland, E. B. (1999). Activation of the CAMP signaling pathway increases apoptosis in human B-precursor cells and is associated with downregulation of Mcl-1 expression. Journal of Cellular Physiology, 180(1):71-80.

Ni, S. and Vingron, M. (2012). R2KS: a novel measure for comparing gene expression based on ranked gene lists. Journal of Computational Biology, 19(6):766-775.

Paredes, J., Figueiredo, J., Albergaria, A., Oliveira, P., Carvalho, J., Ribeiro, A. S., Caldeira, J., Costa, Â. M., Simões-Correia, J., Oliveira, M. J., Pinheiro, H., Pinho, S. S., Mateus, R., Reis, C. A., Leite, M., Fernandes, M. S. F., Schmitt, F., Carneiro, F., Figueiredo, C., Oliveira, C., and Seruca, R. (2012). Epithelial E-and P-cadherins: role and clinical significance in cancer. Biochimica et Biophysica Acta (BBA)-Reviews on Cancer, 1826(2):297-311.

Plaisier, S. B., Taschereau, R., Wong, J. A., and Graeber, T. G. (2010). Rank-rank hypergeometric overlap: identification of statistically significant overlap between gene-expression signatures. Nucleic Acids Research, 38(17):e169-e169.

Proto, M. C., Gazzerro, P., Di Croce, L., Santoro, A., Malfitano, A. M., Pisanti, S., Laezza, C., and Bifulco, M. (2012). Interaction of endocannabinoid system and steroid hormones in the control of colon cancer cell growth. Journal of Cellular Physiology, 227(1):250-258.

Royo, F., Zuñiga-Garcia, P., Torrano, V., Loizaga, A., Sanchez-Mosquera, P., Ugalde-Olano, A., González, E., Cortazar, A. R., Palomo, L., Fernández-Ruiz, S., Lacasa-Viscasillas, I., Berdasco, M., Sutherland, J. D., Barrio, R., Zabala-Letona, A., Martín-Martín, N., Arruabarrena-Aristorena, A., Valcarcel-Jimenez, L., Caro-Maldonado, A., Gonzalez-Tampan, J., Cachi-Fuentes, G., Esteller, M., Aransay, A. M., Unda, M., Falc "®n-Pérez, J. M., and Carracedo, A. (2016). Transcriptomic profiling of urine extracellular vesicles reveals alterations of CDH3 in prostate cancer. Oncotarget, 7(6):6835.

Sanchez-Vega, F., Mina, M., Armenia, J., Chatila, W. K., Luna, A., La, K. C., Dimitriadoy, S., Liu, D. L., Kantheti, H. S., Saghafinia, S., Chakravarty, D., Daian, F., Gao, Q., Bailey, M. H., Liang, W.-W., Foltz, S. M., Shmulevich, I., Ding, L., Heins, Z., Ochoa, A., Gross, B., Gao, J., Zhang, H., Kundra, R., Kandoth, C., Bahceci, I., Dervishi, L., Dogrusoz, U., Zhou, W., Shen, H., Laird, P. W., Way, G. P., Greene, C. S., Liang, H., Xiao, Y., Wang, C., Iavarone, A., Berger, A. H., Bivona, T. G., Lazar, A. J., Hammer, G. D., Giordano, T., Kwong, L. N., McArthur, G., Huang, C., Tward, A. D., Frederick, M. J., McCormick, F., Meyerson, M., Cancer Genome Atlas Research Network; Allen, E. M. V., Cherniack, A. D., Ciriello, G., Sander, C., and Schultz, N. (2018). Oncogenic signaling pathways in the cancer genome atlas. Cell, 173(2):321-337.

Sell, S. and Dunsford, H. (1989). Evidence for the stem cell origin of hepatocellular carcinoma and cholangiocarcinoma. The American Journal of Pathology, 134(6):1347.

Serra, F., Romualdi, C., and Fogolari, F. (2016). Similarity measures based on the overlap of ranked genes are effective for comparison and classification of microarray data. Journal of Computational Biology, 23(7):603-614.

Shi, X., Yi, H., and Ma, S. (2014). Measures for the degree of overlap of gene signatures and applications to TCGA. Briefings in Bioinformatics, 16(5):735-744.

Shureiqi, I., Jiang, W., Zuo, X., Wu, Y., Stimmel, J. B., Leesnitzer, L. M., Morris, J. S., Fan, H.-Z., Fischer, S. M., and Lippman, S. M. (2003). The 15-lipoxygenase-1 product 13-S-hydroxyoctadecadienoic acid down-regulates PPAR- $\delta$ to induce apoptosis in colorectal cancer cells. Proceedings of the National Academy of Sciences, 100(17):9968-9973.

Sousa, B., Ribeiro, A. S., Nobre, A. R., Lopes, N., Martins, D., Pinheiro, C., Vieira, A. F., Albergaria, A., Gerhard, R., Schmitt, F., Baltazar, F., and Paredes, J. (2014). The basal epithelial marker P-cadherin associates with breast cancer cell populations harboring a glycolytic and acid-resistant phenotype. $B M C$ Cancer, 14(1):734.

Subramanian, A., Tamayo, P., Mootha, V. K., Mukherjee, S., Ebert, B. L., Gillette, M. A., Paulovich, A., Pomeroy, S. L., Golub, T. R., Lander, E. S., and Mesirov, J. P. (2005). Gene set enrichment analysis: a knowledge-based approach for interpreting genome-wide expression profiles. Proceedings of the National Academy of Sciences, 102(43):15545-15550.

Sun, L., Hu, H., Peng, L., Zhou, Z., Zhao, X., Pan, J., Sun, L., Yang, Z., and Ran, Y. (2011). P-cadherin promotes liver metastasis and is associated with poor prognosis in colon cancer. The American Journal 
of Pathology, 179(1):380-390.

Sutherland, K. D., Song, J.-Y., Kwon, M. C., Proost, N., Zevenhoven, J., and Berns, A. (2014). Multiple cells-of-origin of mutant K-Ras-induced mouse lung adenocarcinoma. Proceedings of the National Academy of Sciences, 111(13):4952-4957.

Thorsson, V., Gibbs, D. L., Brown, S. D., Wolf, D., Bortone, D. S., Yang, T.-H. O., Porta-Pardo, E., Gao, G. F., Plaisier, C. L., Eddy, J. A., Ziv, E., Culhane, A. C., Paull, E. O., Sivakumar, I. K. A., Gentles, A. J., Malhotra, R., Farshidfar, F., Colaprico, A., Parker, J. S., Mose, L. E., Vo, N. S., Liu, J., Liu, Y., Rader, J., Dhankani, V., Reynolds, S. M., Bowlby, R., Califano, A., Cherniack, A. D., Anastassiou, D., Bedognetti, D., Mokrab, Y., Newman, A. M., Rao, A., Chen, K., Krasnitz, A., Hu, H., Malta, T. M., Noushmehr, H., Pedamallu, C. S., Bullman, S., Ojesina, A. I., Lamb, A., Zhou, W., Shen, H., Choueiri, T. K., Weinstein, J. N., Guinney, J., Saltz, J., Holt, R. A., Rabkin, C. S., Cancer Genome Atlas Research Network; Lazar, A. J., Serody, J. S., Demicco, E. G., Disis, M. L., Vincent, B. G., and Shmulevich, I. (2018). The immune landscape of cancer. Immunity, 48(4):812-830.

Van Marck, V., Stove, C., Jacobs, K., Van den Eynden, G., and Bracke, M. (2011). P-cadherin in adhesion and invasion: Opposite roles in colon and bladder carcinoma. International Journal of Cancer, 128(5):1031-1044.

Vieira, A. F., Dionísio, M. R., Gomes, M., Cameselle-Teijeiro, J. F., Lacerda, M., Amendoeira, I., Schmitt, F., and Paredes, J. (2017). P-cadherin: a useful biomarker for axillary-based breast cancer decisions in the clinical practice. Modern Pathology, 30(5):698.

Von Mering, C., Jensen, L. J., Kuhn, M., Chaffron, S., Doerks, T., Krüger, B., Snel, B., and Bork, P. (2006). STRING 7-ecent developments in the integration and prediction of protein interactions. Nucleic Acids Research, 35(suppl_1):D358-D362.

Wormald, S., Milla, L., and O'Connor, L. (2013). Association of candidate single nucleotide polymorphisms with somatic mutation of the epidermal growth factor receptor pathway. BMC Medical Genomics, 6(1):43.

Xu, B., Goldman, J., Rymar, V., Forget, C., Lo, P., Bull, S., Vereker, E., Barker, P., Trudeau, L., Sadikot, A., and Kennedy, T. E. (2010). Critical roles for the netrin receptor deleted in colorectal cancer in dopaminergic neuronal precursor migration, axon guidance, and axon arborization. Neuroscience, 169(2):932-949.

Yang, W.-L. and Frucht, H. (2001). Activation of the PPAR pathway induces apoptosis and COX-2 inhibition in HT-29 human colon cancer cells. Carcinogenesis, 22(9):1379-1383.

Yang, X., Bentink, S., Scheid, S., and Spang, R. (2006). Similarities of ordered gene lists. Journal of Bioinformatics and Computational Biology, 4(03):693-708. 
Figure 1

Figure 1

Powers of EucD, FES_0.001, FES_0.01, OrderedList, SimSIP and WeiSumE* when $0=1$ with 12 scenarios.

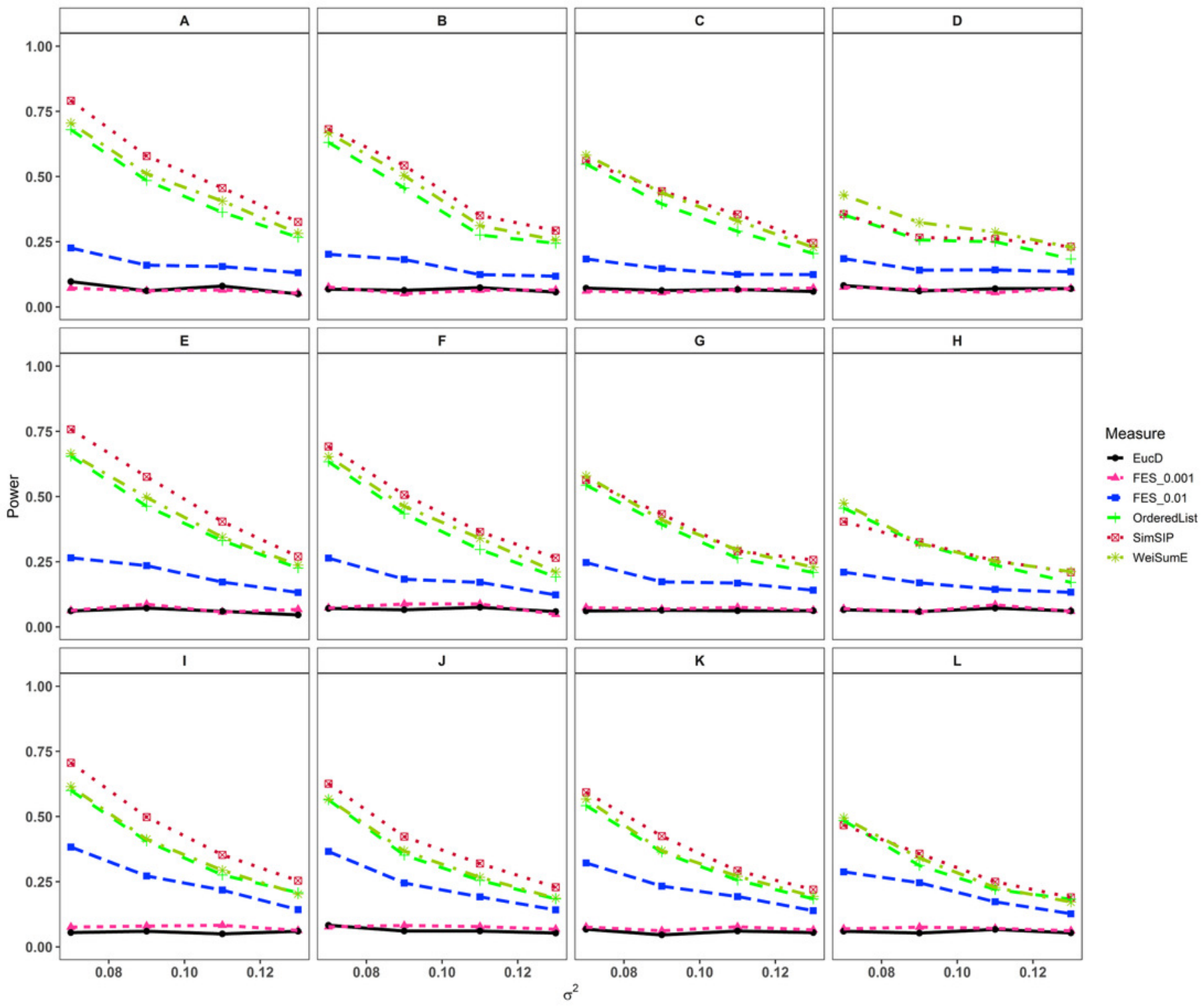


Figure 2

Figure 2

Powers of EucD, FES_0.001, FES_0.01, OrderedList, SimSIP and WeiSumE* when $0=5$ with 9 scenarios.

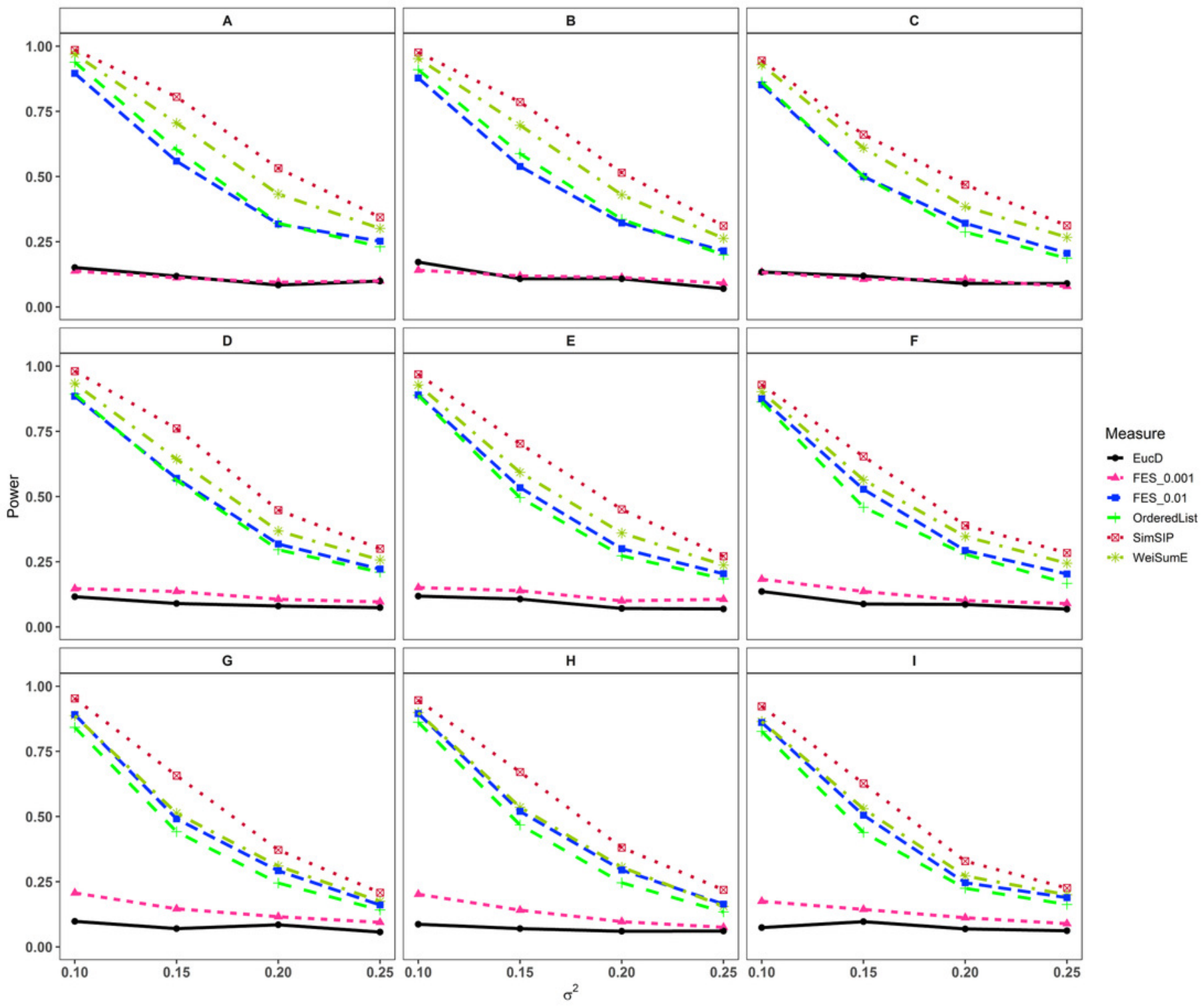


Figure 3

Figure 3

The empirical $p$ values of SimSIP (black dot) and WeiSumE* (red square) for 81 cancer pairs found with both SimSIP and WeiSumE*

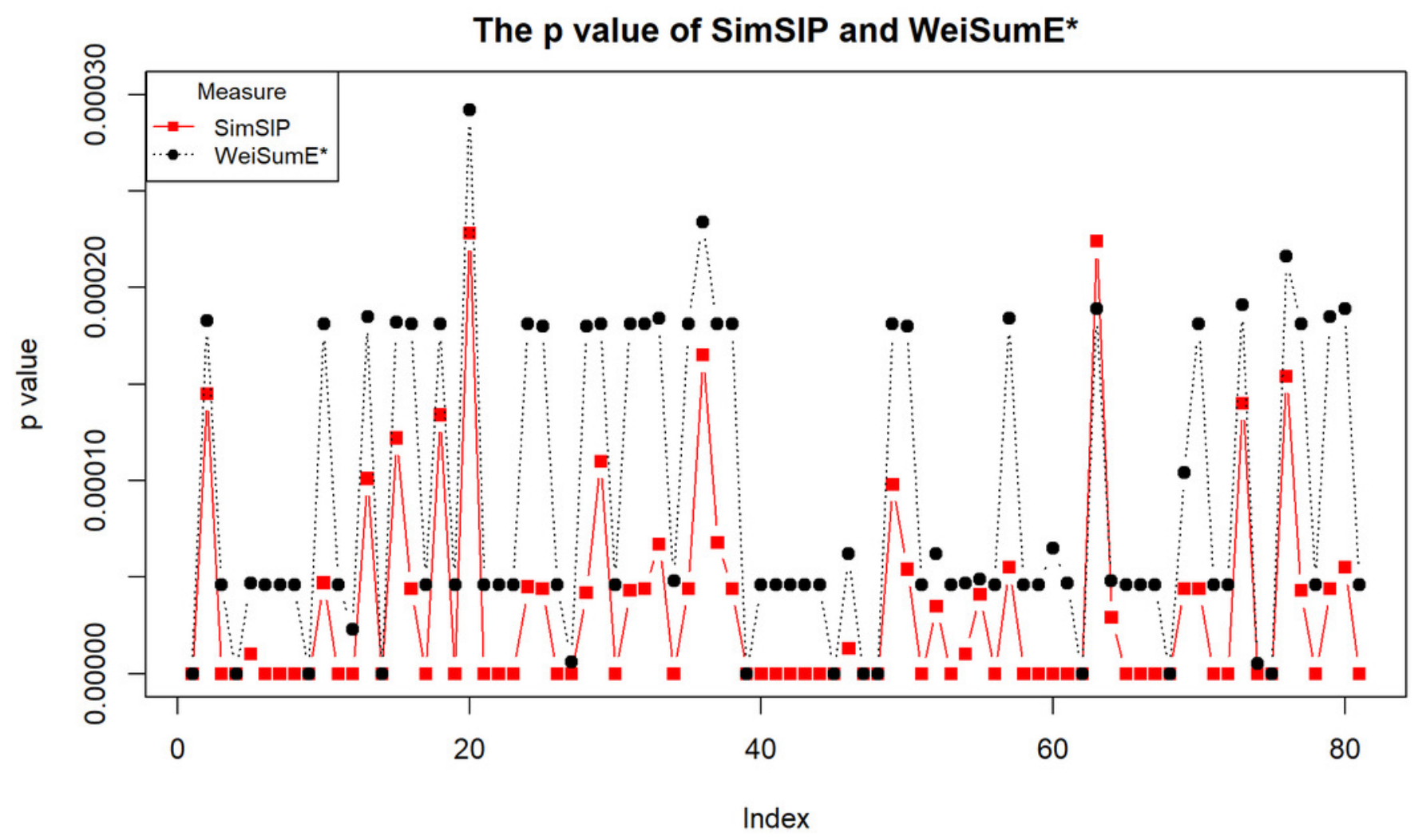


Figure 4

Figure 4

The diseases network of 18 cancer types.

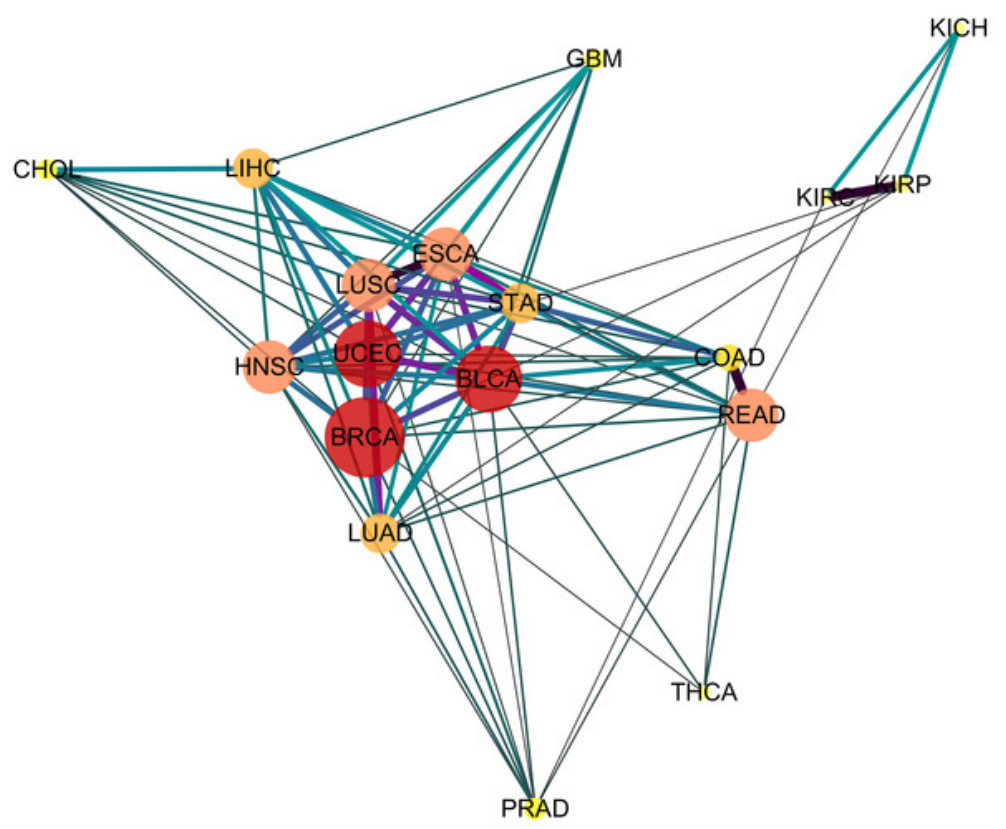

